\title{
The Microwave Energy Harvesting by Absorbent Structures Based on Composite Materials
}

\author{
J. Dikun, V. Jankunas, E. Guseinoviene, A. Senulis, T.C. Akinci
}

\begin{abstract}
This study presents two types of Salesbary Screen models which can be used for the microwave EMR harvesting in the occupational areas for the prevention case of the EMF impact for human health. The proposal structures operate on the 6$18 \mathrm{GHz}$ frequency electromagnetic radiation and allow collecting heating energy of the defined range. There are two types of fillers of $3 \%$ MWCNTs and $3 \%$ CNFs where considered in meaning of well electromagnetic radiation absorbency properties. The special attention had been paid for the outer, spacer and back layers thickness evaluation. The specific power as well as the total power in the proposal structures were calculated.
\end{abstract}

Index Terms - Radar absorbent materials; Electromagnetic radiation; Energy harvesting.

\section{INTRODUCTION}

$\mathrm{E}$ LECTROMAGNETIC fields (EMF) are presented everywhere in our environment but they are invisible and insensitive to the human. Electromagnetic radiation (EMR) depending on wave's length or frequency is divided by several ranges [1]. Nowadays the exposure of EMR for the human health is well studied [2], [3]. The EMR effect level for human body and tissues strongly depends on electromagnetic waves (EMW) frequency or, in other words, on the wave length as well as on the time duration in the EMF. However, the most harmful effects are applied by the high frequency electromagnetic waves (HFEMW). HFEMW are radiated by various kinds of higher frequency radio waves using to transmit the information weather via TV antennas, radio stations or mobile phone base stations can dramatically affect for the human health [3], [4], [5] and [6]. The time limitation under HFEMW is defined by World Health Organization and it must not exceed 6 min [7]. People can be radiated for short time by HFEMW in open or general areas.

J. DIKUN, is with Department of Electrical Engineering University of Klaipeda, Klaipeda, Lithuania, (e-mail: jeldik@bk.ru).

V. JANKUNAS, is with Department of Electrical Engineering University of Klaipeda, Klaipėda, Lithuania, (e-mail: valdas.jankunas@hotmail.com).

E. GUSEINOVIENE, is with Department of Electrical Engineering University of Klaipeda, Klaipedda, Lithuania, (e-mail: eleonora.guseinoviene@ku.lt).

A. SENULIS, is with Department of Electrical Engineering University of Klaipeda, Klaipėda, Lithuania, (e-mail: audrius.senulis@ku.lt).

T.C. AKINCI is with Department of Electrical \& Electronics Engineering, Kirklareli University, Kirklareli, Turkey, (e-mail: cetinakinci@ hotmail.com).
This exposure can be neglected if the sources of HFEMW are sufficiently far from the defined areas Nevertheless; there are a lot of occupational areas where the workers, operators and technical staff are under direct contact with the broadcasting and communications fields in the form of cellular telephones and towers. This contact is possible in the health care industry for medical treatment, in the food industry for the processing and cooking of food, in the wood, textile, and glass fiber industries for drying materials as well as in the automotive, electrical, rubber, and plastic products industries for fusing and sealing operations. The scientific researches indicate a relationship between exposure to microwave radiation and birth defects, such as mongolism (Down's syndrome) and central nervous system damage. The HFEMW may also cause damage to the male testis genital organs [3], [5], [6]. For the prevention of damage to human health the radar absorbing material and structures should be used. This study presents the radar absorbing structure based on the Salesbary Screen (SS) for the EMR heat energy harvesting. The proposal model of SS (Fig.1) consists of a simple structure of thin resistive graphite film, a dielectric spacer layer, and a backside metal reflector. In terms of transmission line theory, the front resistive sheet is placed a quarter wavelengths away from the metallic backside reflector in this structure.

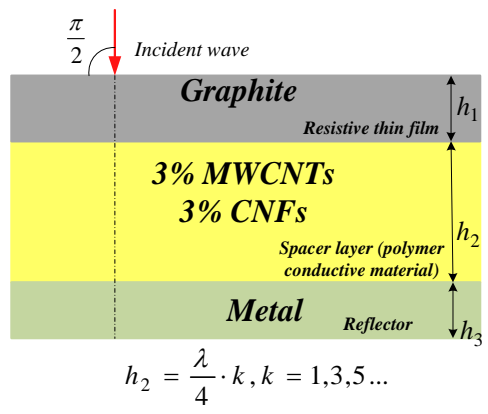

a)

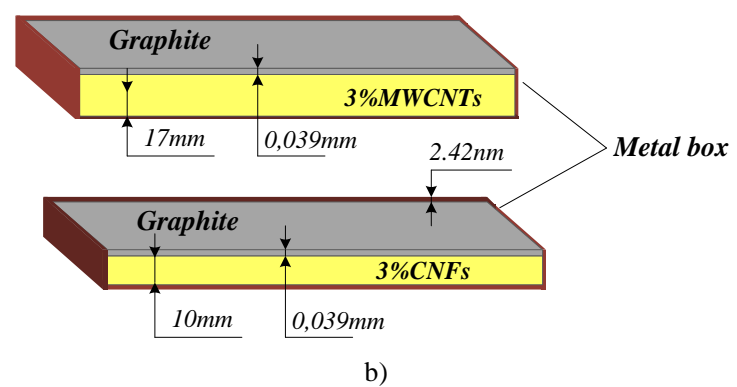

Fig.1. Proposal structure of Salesbary Screen 
According to [8] the best reflection is achieved when incident angle of the wave is 900 and the distance between resistive thin film and reflector is smaller. Also the worst reflection occurs with 377 Ohms resistance sheet. Despite the fact that there are a lot of new composite fillers, to assess the absorbent capacity of SS in these study composite fillers, which electrical parameters had been well studied in a certain frequency range, were used [9]. The dielectric permittivity, magnetic permeability, electrical conductivity and optical refractive index of most sample as well as composites fillers are defined as a function of frequency. And, they are complex valued quantities, which can be established only empirically by measurements [10]. For this reason of the proposal absorbing structure, mathematical modelling of the composite materials based on different weight (wt) percentage of carbon nanopowders with respect to the epoxy resin have been analyzed. Particularly, wt of 3\% for MWCNTs (Multiwall Carbon Nanotubes) based composite materials and wt of 3\% for CNFs (Carbon Nanofibers) based composite materials. For two composite fillers as well as graphite, real and imaginary parts of dielectric permittivity variation on 6$18 \mathrm{GHz}$ frequency range are shown in Table 1.

TABLE I

REAL AND IMAGINARY COMPONENT OF PERMITTIVITY OF NANOSTRUCTURED COMPOSITE MATERIALS AND GRAPHITE UNDER THE 6 $18 \mathrm{GHz}$ FREQUENCY BAND [9],[10]

\begin{tabular}{|c|c|c|c|c|c|c|c|c|c|c|c|c|c|c|}
\hline \multirow{3}{*}{ Type of fillers } & \multicolumn{14}{|c|}{ Frequency, GHz } \\
\hline & \multicolumn{2}{|c|}{6} & \multicolumn{2}{|c|}{8} & \multicolumn{2}{|c|}{10} & \multicolumn{2}{|c|}{12} & \multicolumn{2}{|c|}{14} & \multicolumn{2}{|c|}{16} & \multicolumn{2}{|c|}{18} \\
\hline & $\varepsilon_{r}$ & $\varepsilon_{r}^{\prime \prime}$ & $\varepsilon_{r}^{\prime}$ & $\varepsilon_{r}^{\prime \prime}$ & $\varepsilon_{r}$ & $\varepsilon_{r}^{\prime \prime}$ & $\varepsilon_{r}^{\prime}$ & $\varepsilon_{r}^{\prime \prime}$ & $\varepsilon_{r}^{\prime}$ & $\varepsilon_{r}^{\prime \prime}$ & $\varepsilon_{r}^{\prime}$ & $\varepsilon_{r}^{\prime \prime}$ & $\varepsilon_{r}^{\prime}$ & $\varepsilon_{r}^{\prime \prime}$ \\
\hline $3 \%$ MWCNTs & 18.1 & 14.5 & 17.8 & 14.1 & 17.4 & 13.8 & 17.2 & 13.6 & 17 & 13.4 & 16.8 & 13.3 & 16.6 & 13.1 \\
\hline $3 \% \quad$ CNFs & 14.7 & 5.2 & 13.6 & 5.3 & 12.9 & 5.4 & 12.3 & 5.5 & 11.8 & 5.55 & 11.4 & 5.6 & 11 & 5.7 \\
\hline graphite & 13.5 & 8.1 & 12.2 & 7.8 & 11.1 & 7.0 & 10.7 & 6.6 & 10.1 & 6.4 & 9.8 & 6.2 & 9.5 & 6.0 \\
\hline
\end{tabular}

\section{MATHEMATICAL BACKGROUND}

With the reason of collecting the more energy in the proposal structure, the precise calculation of layers heights is necessary. The EMW propagation through the different materials is based on optics laws [14], [15]. The task of the energy harvesting by SS means that the incident EMW has freely propagate through the first and second layers and has not transmitted through the third layer. In this case the reflected wave from the back layer returns to the medium and outer layers and goes out beyond the structure. The resonance should be achieved in the spacer layer for the energy collecting.

\subsection{The model layers thickness calculation}

In this chapter, the outer, spacer and back layers thicknesses had been obtained.

\subsubsection{Resistive sheet thickness}

For the wave that freely passed through the graphite layer, it is required that its resistivity would be equal to the air resistivity, namely $377 \Omega$. Outer layer' impedance can be calculated:

$$
\frac{Z_{\mathrm{m}}}{Z_{\text {air }}}=\sqrt{\frac{\mu_{y}^{*}}{\varepsilon_{y}^{*}}}
$$

Where: $Z_{m}-$ is medium impedance, $[\Omega]$;

$Z_{\text {air }}-$ is the air impedance, $[\Omega] ; Z_{\text {air }}=377 \Omega$;

$\mu_{\gamma}^{*}$ and $\varepsilon_{\%}^{*}-$ are the complex permeability and

permittivity respectively.

Taking in account graphite diamagnetic properties it is assumed that its permeability is equal to 1. Therefore the Eq. (1) can be presented as:

$Z_{\mathrm{m}}=\frac{377}{\sqrt{\varepsilon_{\%}^{*}}}$

The complex permittivity is calculated:

$$
\varepsilon_{Y}^{*}=\frac{\varepsilon^{s}-i \cdot \varepsilon^{n s}}{\varepsilon_{0}}
$$

Here: $\varepsilon_{\gamma}^{*}-$ is the relative permittivity of the medium directly contacted with air;

$\varepsilon^{v}-$ is the real part of complex permittivity;

$\varepsilon^{\sharp s}$ - is the imaginary part of complex permittivity;

$\varepsilon_{0}-$ is dielectric constant.

As a graphite layer thickness the depth $\mathrm{h}_{1}$ should be calculated according to the skin depth for non-metals at high frequencies formula [12]:

$$
h_{1}=d(f)=\frac{c \cdot \sqrt{\varepsilon_{\gamma}}}{f \times \varepsilon_{\sigma}^{s y}}
$$

Where: $c-$ is light speed in vacuum, $[\mathrm{m} / \mathrm{s}]$;

$$
\begin{aligned}
& f-\text { is frequency, }[\mathrm{Hz}] \\
& d(f)-\text { is skin depth as function of frequency, }[\mathrm{m}] .
\end{aligned}
$$

\subsubsection{Spacer layers thickness}

In respect that proposal structure should absorb all $6-18 \mathrm{GHz}$ frequencies range EMW, it is needed to evaluate the skin depth for the MWCNTs and CNFs composites under defined frequency range, and as the spacer layer height the maximum penetration depth must be considered as:

$h_{2}=d(f)_{\max }$

The relative permeability of the composites is also equal to 1, therefore, for the spacer layer, thickness evaluation the equation (4) can be used. For the resonance which is the key to successful absorption it is necessary that the number of wave length quarter must be equal to odd number in the spacer layer. The number of wave length quarter is calculated as:

$\tau=\frac{d(f)_{\max }}{\frac{\lambda_{i}}{4}}$

Here: $\tau-$ is the quarter number of wave length; 
$i-$ is the certain frequency wave length index.

Wave length in the composites medium is calculated as:

$$
\lambda=\frac{e}{f \sqrt{\varepsilon_{r}}} .
$$

Complex permittivity is calculated by following equation:

$$
\varepsilon_{\gamma}=\sqrt{\left(\varepsilon_{\gamma}^{b}\right)^{2}+\left(\varepsilon_{\gamma}^{s p}\right)^{2}} .
$$

Where: $\varepsilon_{y}^{s}$ and $\varepsilon_{\gamma}^{s s}$ - are the real and imaginary parts of relative permittivity accordingly.

\subsubsection{Back layer thickness}

EMW cannot penetrate from the metal surface with thickness which is larger than the skin depth. For this reason the thickness of back layer is calculated as:

$$
h_{a}>d_{M \theta}(f)=\sqrt{\frac{1}{\pi f \mu \sigma}},
$$

Here: $\sigma-$ is the electric conductivity, $[\mathrm{S} / \mathrm{m}]$.

\subsection{Absorbance factor calculation}

Taking in account that for polymer conductive material, the permeability is equal to 1 , the equations of absorbency factors are:

$$
\alpha=1-\left|\frac{\sqrt{\varepsilon} \cdot\left(1+j \cdot \operatorname{ctg}\left(k h_{2}\right)\right)-\frac{1}{\rho_{r g}}}{\sqrt{\varepsilon} \cdot\left(1-j \cdot \operatorname{ctg}\left(k h_{2}\right)\right)+\frac{1}{\rho_{r g}}}\right|^{2}
$$

Where: $k$ - is wave number, $\left[\mathrm{m}^{-1}\right]$;

$h_{2}$ - is the thickness of dielectric layer (polymer conductive material), [m];

$$
\rho_{g}-\text { is the resistivity of outer layer, }[\Omega \mathrm{m}] \text {. }
$$

Wave number is calculated as:

$$
k=2 \pi f \sqrt{\varepsilon \mu_{0} \varepsilon_{0}}=2.1 \cdot 10^{-8} f \sqrt{\varepsilon} .
$$

The resistivity of dielectrics namely of outer layer can be represented by conductivity which is the frequency function:

$$
\sigma=2 \pi f \varepsilon_{0} \varepsilon_{y}^{s p} \approx 55,63 \cdot 10^{-12} f \varepsilon_{\gamma}^{s s}
$$

Here: $\sigma-$ is the electric conductivity $[\mathrm{S} / \mathrm{m}]$.

Therefore formula (10) can be rewriten as:

$\alpha=1-\left|\frac{\sqrt{\varepsilon} \cdot\left(1+j \cdot \operatorname{ctg}\left(k h_{2}\right)\right)-\sigma_{r s}}{\sqrt{\varepsilon} \cdot\left(1-j \cdot \operatorname{ctg}\left(k h_{2}\right)\right)+\sigma_{r s}}\right|^{2}$

\section{II.3. Power calculation}

This chapter presents the specific power index as well as total power calculation results for the proposal structures.

\section{II.3.1 Specific power factor calculation}

Specific power released in the form of heat per unit volume in material is determined by the following relationship:

$$
P_{S}=5.55 \cdot \varepsilon_{y} \cdot \operatorname{tg} \varphi \cdot f \cdot E^{2} \times 10^{-11},
$$

Where: $P_{S}-$ is specific power, $\left[\mathrm{W} / \mathrm{m}^{3}\right]$;

$E$ - is electric field strength inside of medium; [V/m], $\operatorname{tg} \varphi$-is the loss tangent.

Loss tangent is defined as the ratio:

$$
\operatorname{tg} \varphi=\frac{z_{r}^{n}}{\varepsilon_{\sigma}^{g}} .
$$

Intrinsic electric field in interested layer could be obtained by using the following formula:

$$
E=\frac{E_{0}}{\varepsilon_{v_{q r}} \varepsilon_{r_{s I}}},
$$

Here: $E_{0}-$ is external electrical field strength, $[\mathrm{V} / \mathrm{m}]$;

$\varepsilon_{v_{g r}}-$ is relative permittivity of graphite;

$\varepsilon_{v_{g l}}-$ is relative permittivity of the spacer layer's material.

\subsubsection{The structure total power calculation}

Total power can be calculated as:

$$
P=P_{5} \cdot V=P_{5} \cdot h \cdot l \cdot w_{x}
$$

Where: $h_{s} l_{w} w$ - structure's height, length and width respectively, [m].

\section{Calculations Results}

Here the layers thicknesses and power calculation results are presented.

\section{III.1 Layers' thicknesses}

The layers thicknesses had been given as shown in Table 2 . Calculation results are presented in the Tables 2 and 3:

TABLE II

GRAPHITE LAYER PROPERTIES CALCULATION RESULTS

\begin{tabular}{c|cccc}
$f,[\mathbf{G H z}]$ & $\varepsilon_{r}$ & $d(f),[m]$ & $Z$, & $\sigma,[\mathbf{S} / \mathbf{m}]$ \\
& & & {$[\mathbf{\Omega}]$} & \\
\hline $\mathbf{6}$ & 15.66 & 0.024 & 286 & 2.70 \\
$\mathbf{8}$ & 14.48 & 0.018 & 297 & 3.47 \\
$\mathbf{1 0}$ & 13.21 & 0.016 & 311 & 3.89 \\
$\mathbf{1 2}$ & 12.57 & 0.013 & 319 & 4.41 \\
$\mathbf{1 4}$ & 12.04 & 0.012 & 326 & 4.98 \\
$\mathbf{1 6}$ & 11.60 & 0.010 & 332 & 5.52 \\
$\mathbf{1 8}$ & 11.32 & $\mathbf{0 . 0 0 9}$ & 336 & 6.01
\end{tabular}

As it is shown in Table 2, the impedance of $336 \Omega$ under the frequency of $18 \mathrm{GHz}$ is very close to the air impedance. Therefore the outer layer thickness should be defined as equal to $9 \mathrm{~mm}$. However it is necessary to evaluate the absorbency factor for this layer under the applied frequency range of 618GHz. Calculation had been done using Equations (9) - (12). These results are presented in the Table 3 . 
TABLE III

ABSORPTANCE AND TRANSMITTANCE FACTORS FOR OUTER LAYER

\begin{tabular}{|c|c|c|c|c|c|c|c|c|c|}
\hline \multirow{2}{*}{$\begin{array}{c}h_{1}=\mathrm{d}(f) \\
,[\mathrm{m}]\end{array}$} & \multicolumn{7}{|c|}{$f,[\mathrm{GHz}]$} & \multirow[t]{2}{*}{$\begin{array}{l}\text { Average value of } \\
\text { absorptance }\end{array}$} & \multirow[t]{2}{*}{$\begin{array}{l}\text { Average value of } \\
\text { transmittance }\end{array}$} \\
\hline & 6 & 8 & 10 & 12 & 14 & 16 & 18 & & \\
\hline 0.024 & 0.54 & 0.29 & 0.50 & 0.52 & 0.63 & 0.76 & 0.82 & 0.58 & 0.42 \\
\hline 0.018 & 0.38 & 0.87 & 0.92 & 0.31 & 0.46 & 0.97 & 0.82 & 0.68 & 0.32 \\
\hline 0.016 & 0.96 & 0.73 & 0.28 & 0.97 & 0.60 & 0.52 & 0.98 & 0.72 & 0.28 \\
\hline 0.013 & 0.10 & 0.89 & 0.46 & 0.84 & 0.67 & 0.78 & 0.78 & 0.65 & 0.35 \\
\hline 0.012 & 0.20 & 0.96 & 0.18 & 0.95 & 0.26 & 0.93 & 0.42 & 0.56 & 0.44 \\
\hline 0.010 & 0.93 & 0.03 & 0.96 & 0.46 & 0.74 & 0.92 & 0.07 & 0.59 & 0.41 \\
\hline 0.009 & 0.94 & 0.48 & 0.55 & 0.97 & 0.16 & 0.81 & 0.92 & 0.69 & 0.31 \\
\hline
\end{tabular}

The results of Table 3 indicate that the most suitable outer layer thickness is $12 \mathrm{~mm}$, because for this chosen value the average absorbency factor under the applied frequency range is the lowest, namely 0.56 ; and as a result the transmission factor through the graphite layer is highest, namely 0.44 . It means that only $44 \%$ incident EMW with different length would be penetrating to resistive sheet. For the effectively energy collection by proposal structures it is necessary to evaluate the graphite layer height such that the transmission factor would be approaching to 1 . Therefore, Table 4 presents calculations of the most appropriate outer layer height. As it is seen, average value of the highest transmittance occurred if the graphite layer height is equal $0.039 \mathrm{~mm}$.

TABLE IV

OUTER LAYER THICKNESS EVAULATION RESULTS

\begin{tabular}{|c|c|c|c|c|c|c|c|c|c|}
\hline \multicolumn{2}{|c|}{ Estimated height $\mathrm{h}_{1},[\mathrm{~mm}]$} & 5.000 & 2.500 & 1.250 & 0.625 & 0.313 & 0.156 & 0.078 & 0.039 \\
\hline$f,[\mathrm{GHz}]$ & $\lambda,[\mathrm{mm}]$ & \multicolumn{8}{|c|}{ Transmittance factors } \\
\hline 6 & 12.63 & 0.3318 & 0.9654 & 0.9864 & 0.7319 & 0.9198 & 0.9789 & 0.9947 & 0.9987 \\
\hline 8 & 9.85 & 0.9895 & 0.9900 & 0.9778 & 0.6136 & 0.8733 & 0.9657 & 0.9912 & 0.9978 \\
\hline 10 & 8.25 & 0.3081 & 0.9629 & 0.9686 & 0.5149 & 0.8271 & 0.9517 & 0.9875 & 0.9969 \\
\hline 12 & 7.05 & 0.0210 & 0.8503 & 0.9574 & 0.4214 & 0.7750 & 0.9347 & 0.9830 & 0.9957 \\
\hline 14 & 6.18 & 0.0486 & 0.6130 & 0.9451 & 0.3417 & 0.7222 & 0.9163 & 0.9779 & 0.9944 \\
\hline 16 & 5.51 & 0.4182 & 0.2270 & 0.9317 & 0.2745 & 0.6698 & 0.8965 & 0.9724 & 0.9930 \\
\hline 18 & 4.95 & 0.9800 & 0.0051 & 0.9167 & 0.2162 & 0.6165 & 0.8746 & 0.9660 & 0.9913 \\
\hline \multicolumn{2}{|c|}{$\frac{10}{\text { Average value of transmittance }}$} & 0.4425 & 0.6591 & 0.9548 & 0.4449 & 0.7720 & 0.9312 & 0.9818 & 0.9954 \\
\hline
\end{tabular}

For the evaluation of absorbency of middle structures layers, properties of composites, calculation results for 3\% MWCNTs the wave lengths and thickness had been calculated. For the and 3\% CNFs fillers are shown in Tables 5 and 6.

TABLE V

COMPOSITES FILLERS ELECTRICAL PROPERTIES

\begin{tabular}{|c|c|c|c|c|c|c|c|c|}
\hline & \multicolumn{5}{|c|}{$3 \%$} & \multicolumn{4}{|c|}{$3 \%$ CNFs } \\
\hline$f,[\mathrm{GHz}]$ & $\varepsilon_{r}$ & $\mathrm{~d}(f),[\mathrm{m}]$ & $\mathrm{Z},[\Omega]$ & $\sigma,[\mathrm{S} / \mathrm{m}]$ & $\varepsilon_{r}$ & $\mathrm{~d}(f),[\mathrm{m}]$ & $\mathrm{Z},[\Omega]$ & $\sigma,[\mathrm{S} / \mathrm{m}]$ \\
\hline 6 & 23.19 & 0.017 & 78.28 & 4.84 & 15.60 & 0.038 & 95.45 & 1.74 \\
\hline 8 & 22.71 & 0.013 & 79.11 & 6.28 & 14.57 & 0.027 & 98.77 & 2.37 \\
\hline 10 & 22.21 & 0.010 & 80.00 & 7.68 & 14.00 & 0.021 & 100.76 & 3.03 \\
\hline 12 & 21.93 & 0.009 & 80.51 & 9.08 & 13.48 & 0.017 & 102.68 & 3.68 \\
\hline 14 & 21.65 & 0.007 & 81.03 & 10.44 & 13.06 & 0.014 & 104.33 & 4.35 \\
\hline 16 & 21.43 & 0.007 & 81.44 & 11.84 & 12.70 & 0.012 & 105.78 & 4.98 \\
\hline 18 & 21.15 & 0.006 & 81.98 & 13.12 & 12.39 & 0.010 & 107.11 & 5.71 \\
\hline
\end{tabular}

TABLE VI

WAVE LENGTH AND SPACER LAYER THICKNESS CALCULATION RESULTS

\begin{tabular}{|c|c|c|c|c|c|c|c|c|}
\hline \multirow{2}{*}{$f,[\mathrm{GHz}]$} & \multicolumn{4}{|c|}{$3 \%$ MWCNTs } & \multicolumn{4}{|c|}{$3 \%$ CNFs } \\
\cline { 2 - 9 } & $\mathrm{d}(f),[\mathrm{cm}]$ & $\lambda,[\mathrm{cm}]$ & $\lambda / 4,[\mathrm{~cm}]$ & $\tau$ & $\mathrm{d}(f),[\mathrm{cm}]$ & $\lambda,[\mathrm{cm}]$ & $\lambda / 4,[\mathrm{~cm}]$ & $\tau$ \\
\hline 6 & $\mathbf{1 . 7 0 0}$ & 1.037 & 0.26 & 7 & $\mathbf{3 . 8 0 0}$ & 1.266 & 0.316 & 12 \\
\hline 8 & 1.300 & 0.786 & 0.20 & 9 & 2.700 & 0.982 & 0.245 & 16 \\
\hline 10 & 1.000 & 0.637 & 0.16 & 11 & 2.100 & 0.802 & 0.201 & $\mathbf{1 9}$ \\
\hline 12 & 0.900 & 0.534 & 0.13 & 13 & 1.700 & 0.681 & 0.170 & 22 \\
\hline 14 & 0.700 & 0.461 & 0.12 & 15 & 1.400 & 0.594 & 0.148 & 26 \\
\hline 16 & 0.700 & 0.405 & 0.10 & 17 & 1.200 & 0.527 & 0.132 & $\mathbf{2 9}$ \\
\hline 18 & 0.600 & 0.362 & 0.09 & 19 & 1.000 & 0.473 & 0.118 & 32 \\
\hline
\end{tabular}

Presented results show that odd number of quarte wave length very well matches with the depth that is defined as $1.7 \mathrm{~cm}$ for the 3\% MWCNTs filler. However for the 3\% CNFs filler it is not so well. It means that the most possible absorbency factors can be achieved only at frequencies of 8 and $16 \mathrm{GHz}$. With the reason of optimizing this situation, the absorbency factor was 
calculated under the frequency range of $6-18 \mathrm{GHz}$ for all possible thicknesses which are represented in Table 6 . The calculation results are presented in Table 7.

TABLE VII

ABSORBENCY FACTOR FOR 3\% CNFs FILLER LAYER

\begin{tabular}{|c|c|c|c|c|c|c|c|c|}
\hline $\begin{array}{c}h_{2}=\mathrm{d}(f), \\
{[\mathrm{m}]}\end{array}$ & \multicolumn{7}{|c|}{$f,[\mathrm{GHz}]$} & \multirow{2}{*}{ Avrg } \\
\cline { 2 - 10 } & 6 & 8 & 10 & 12 & 14 & 16 & 18 & \\
\hline 0.038 & 0.01 & 0.76 & 0.97 & 0.61 & 0.46 & 0.98 & 0.24 & 0.58 \\
\hline 0.027 & 0.99 & 0.24 & 0.03 & 0.39 & 0.54 & 0.02 & 0.76 & 0.42 \\
\hline 0.021 & 0.01 & 0.76 & 0.97 & 0.61 & 0.46 & 0.98 & 0.24 & 0.57 \\
\hline 0.017 & 0.88 & 0.97 & 0.77 & 0.00 & 0.78 & 0.96 & 0.64 & 0.71 \\
\hline 0.014 & 0.7 & 0.45 & 0.97 & 0.36 & 0.80 & 0.9 & 0.15 & 0.62 \\
\hline 0.012 & 0.27 & 0.98 & 0 & 0.99 & 0.10 & 0.97 & 0.2 & 0.50 \\
\hline 0.001 & 0.5 & 0.66 & 0.77 & 0.85 & 0.91 & 0.95 & 0.97 & $\mathbf{0 . 8 0}$ \\
\hline
\end{tabular}

Finally, the thicknesses of $1.7 \mathrm{~cm}$ and $1.0 \mathrm{~cm}$ were chosen for $3 \%$ MWCNTs and 3\% CNFs fillers of spacer layer. The absorbency factor for them is shown in Fig.1.

SS structure's absorbency factor for different fillers

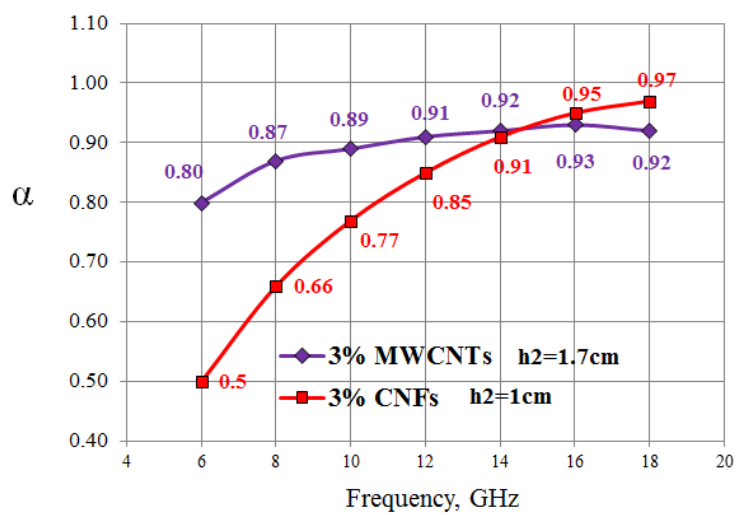

Fig.1.Spacer layers absorbency factor evaluation under 6-18GHz frequency range

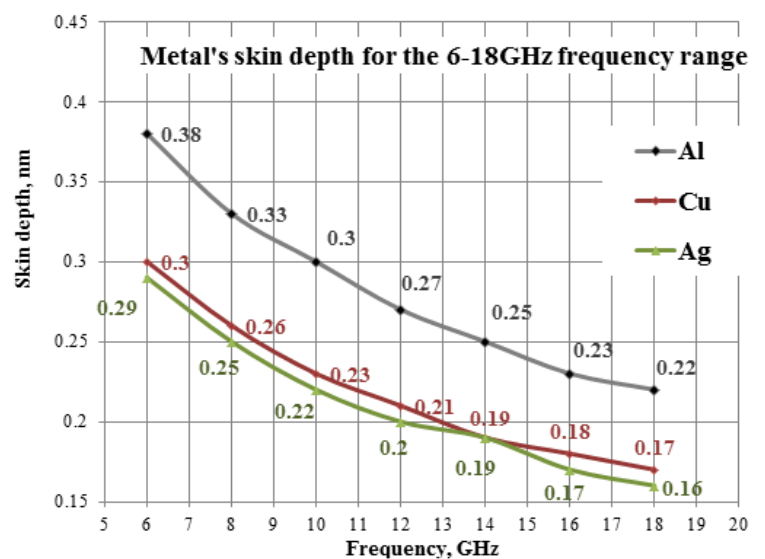

Fig.2. Back layer skin depth evaluation under $6-18 \mathrm{GHz}$ frequency range

In order to evaluate the thickness of back layer there are used three metals namely aliuminium, cooper and silver. For the best shelding ability it is recommended to use of $3 \mathrm{~d}(f), 5 \mathrm{~d}(f)$ and $11 \mathrm{~d}(f)$ for the minimum, medium and exelent shielding respectively [11]. For these three metals the depth skin were calculated under the frequency range of $6-18 \mathrm{GHz}$ assuming the magnetic permeabilities which have the values of $\mu_{\mathrm{r}}=1$ [11]. Calculation results are shown in Fig. 2. Most lower skin depth is appeared for the cooper. The average value of cooper skin depth under applied frequency range is $0.22 \mathrm{~nm}$. Therefore, as it was discussed above, finally the back layer material is choosen cooper and the thikness equal to $2.42 \mathrm{~nm}$.

\section{III.2. Power evaluation}

The specific power factor was calculated using Equations 1416. The external electric field strength with extremely high frequency must not exceed the value of $5 \mathrm{~V} / \mathrm{m}$ in Lithuania [12]. Therefore, for the calculation of specific power factor the outside electric field value of $5 \mathrm{~V} / \mathrm{m}$ was considered. Graphical representation of power factors is shown in Fig. 3.

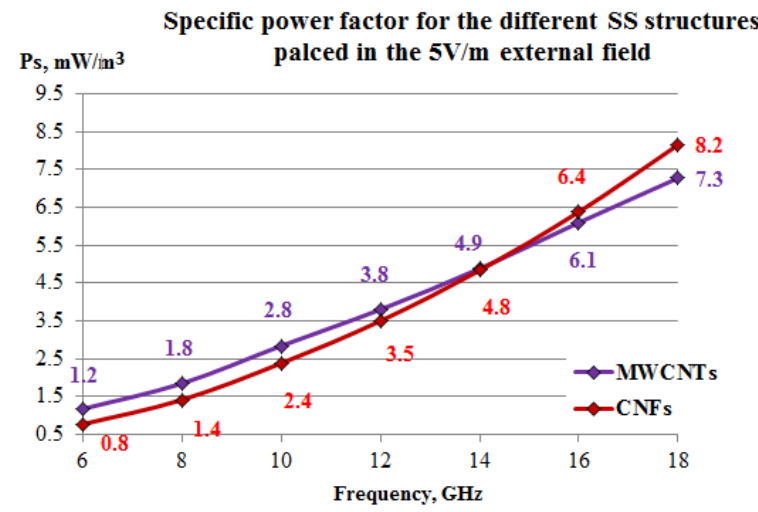

Fig.3. Specific power calculation results

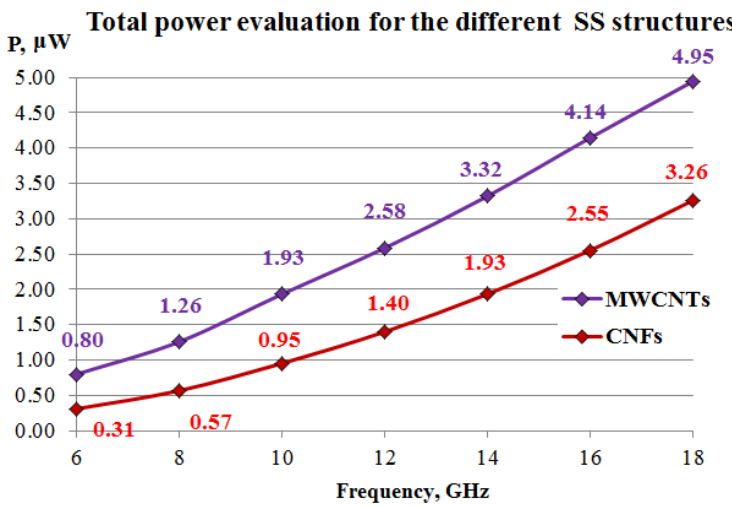

Fig.4. Total power calculation results for the SS structures: $w=l=0,2 m$

Equation (17) defines the total power dependency on the geometrical parameters of spacer layer. It is clear that increasing the structures width and length the total collected power increases. Nevertheless, the precise calculations were done assuming the equality of the length and width. The total collected power in certain volume of the composite material is presented by Fig. 4 .

\section{DISCUSSIONS}

The layers of proposal structures were used because of the data $[9,10]$. Also the graphite as outer layer has very good ability to collect the heating because of its high thermal coefficient that is very relevant in term of further study on the energy converter by these structures. The layers thicknesses 
were obtained to achieve the resonance in the spacer layers which directly influences to the heating properties of composites. Therefore the thicknesses of the proposal structures as well as the spacer layers thicknesses are calculated so that they can operate with all frequencies within the microwave frequency range of $6-18 \mathrm{GHz}$. Average absorbency factor for the $3 \%$ MWCNTs with optimal thickness $1.7 \mathrm{~cm}$ is 0.89 . For the SS with $3 \% \mathrm{CNFs}$ and thickness size of $1 \mathrm{~cm}$ the average absorbency factor is equal to 0.8.The total calculated harvesting power values are very small, average total power for SS with 3\% MWCNTs and with $3 \% \mathrm{CNFs}$ not exceeds the value of $2.71 \mu \mathrm{W}$ and $1.57 \mu \mathrm{W}$ respectively. The main reason is the low value of electric field applied for the calculation. Although the legislation limits of the high frequency electromagnetic field up to $5 \mathrm{~V} / \mathrm{m}$, there are a lot of investigations which find out much more electric field strength values then $5 \mathrm{~V} / \mathrm{m}$. As an example, police's radar system used in speed tacking can radiate from $17 \mathrm{~V} / \mathrm{m}$ to $142 \mathrm{~V} / \mathrm{m}$ for high frequency electromagnetic waves [13]. Therefore, the electric field value of $5 \mathrm{~V} / \mathrm{m}$ could not be assumed as a constant or limit value.

\section{CONCLUDING REMARKS}

In this study, two proposal models with microwave absorbing composites as a spacer layer, graphite resistive sheet and cooper back layer of Salesbary Screen are presented. SS structures provide the best operation which will be possible for only incident electromagnetic waves. The mathematical calculation shows that it is possible to collect electromagnetic microwaves energy. As a further study these types of devices should be constructed and tested in real electromagnetic radiation condition. Also it is needed to evaluate the temperature conditions inside the structure using the thermodynamical physics and obtain the heat exchange processes on the graphite-composite, composite-cooper boundaries. Then it is possible to consider the applying of the Peltier elements for the convert the heating energy to electrical energy by proposal structures. Moreover, the use of such structures is relevant for electromagnetic (EM) protection from natural phenomena (lightning), in nuclear physics for shielding and protection from the nuclear EM pulses; in electromagnetic compatibility (EMC) for equipment-level shielding, for protection from the high-intensity radiated fields (HIRF), and for mitigation of exposure to be considered in human health.

\section{ACKNOWLEDGMENT}

The authors thank Project "Lithuanian Maritime Sector's Technologies and Environment Research Development", Subsidy contract No: VP1-3.1-ŠMM-08-K-01-019, 2012-2015 for the possibility to complete a scientific research.

\section{REFERENCES}

[1] J.G. Van Bladel, Electromagnetic Fields, John Wiley \& Sons, 2007, p.1176.

[2] L. Hardell, C. Sage, "Biological effects from electromagnetic field exposure and public exposure standards", Biomedicine \& pharmacotherapy, Vol.62, No.2, 2008, pp.104-109.
[3] P. Jacob, D. Stram, "Late health effects of radiation exposure: New statistical, epidemiological, and biological approaches", International journal of radiation biology, January 2013, pp.1-11.

[4] I. Calvente, M.F. Fernandez, J. Villalba, N. Olea, M.I. Nunez, "Exposure to electromagnetic fields (non-ionizing radiation) and its relationship with childhood leukemia: A systematic review", Science of the Total Environment; Vol.408, No.16, 2010, pp.3062-3069.

[5] W. Joseph, P. Frei, M. Roosli, G. Thuroczy, P. Gajsek, T. Trcek, J. Bolte, G. Vermeeren, E. Mohler, P. Juhasz, V. Finta, L. Martens, "Comparison of personal radio frequency electromagnetic field exposure in different urban areas across Europe", Environmental Research; Vol.110, No.7, 2010, pp.658-663.

[6] S. Heinrich, S. Thomas, C. Heumann, R. Kries, K. Radon, "The impact of exposure to radio frequency electromagnetic fields on chronic wellbeing in young people--a cross-sectional study based on personal dosimeter", Environment international, Vol.37, No.1, 2011, pp.26-30.

[7] Electromagnetic Fields ( $300 \mathrm{~Hz}$ to $300 \mathrm{GHz}$ ), Environmental Health Criteria 137, World Health Organization, Geneva, Switzerland, 1993.

[8] A.A. Hebeish, M.A. Elgamel, R.A. Abdelhady, A.A. Abdelaziz, "Factors affecting the performance of the radar absorbent textile materials of different types and structures", Progress In Electromagnetics Research B, Vol.3, pp.219-226, 2008.

[9] D. Micheli, C. Apollo, R. Pastore, R.B. Morles, G. Gradoni, M. Marchetti, Chapter of Book: Advances in Nanocomposites - Synthesis, Characterization and Industrial Applications, ISBN 978-953-307-1657, p.966. Title of chapter: Electromagnetic characterization of Composite Materials and Microwave Absorbing Modeling; INTECH Open Access Publisher, 2011.

[10] M. Hotta, M. Hayashi, M.T. Lanagan, D.K. Agrawal, K. Nagata, Complex Permittivity of Graphite, Carbon Black and Coal Powders in the Ranges of X-band Frequencies (8.2 to $12.4 \mathrm{GHz}$ ) and between 1 and $10 \mathrm{GHz}$; ISIJ International, Vol.51, No.11, 2011, pp. 1766-1772.

[11] R.B. Tabrizi, "Electircal and Magnetic Properties of Metals", ASM International, 2011, p.270.

[12] Electromagnetic fields in the occupational and general environment, (2011); The $10 \mathrm{kHz}$ - $300 \mathrm{GHz}$ frequency bands normalized parametersvalues and measurement requirements, HN 80, No.V-199, 2011.

[13] E.D. Mantiply, R.P. Kenneth, S.W. Poppell, J.A. Murphy, "Summary of Measured Radiofrequency Electric and Magnetic Fields (10 kHz to $30 \mathrm{GHz}$ ) in the General and Work Environment", Bio electromagnetics 18, 2007, pp.563-577.

[14] J.A. Stratton, Electromagnetic Theory. Hoboken, NJ: Wiley-IEEE Press, 2007.

[15] P. Saville, Review of Radar Absorbing Materials, Defence R\&D Canada - Atlantic, 2005.

\section{BIOGRAPHIES}

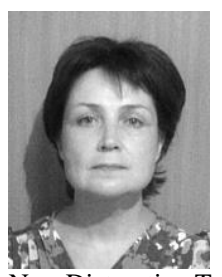

JELENA DIKUN was born in Klaipeda, Lithuania. She received the B.Sc., and M.Sc., degrees from Klaipeda University, in Electrical Engineering Department, in 2011 and 2013 respectively. Currently, she is a Ph.D. student at Prof. K. Barsausko Ultrasound Research Institute of Kaunas Technology University. Her research interests are in Electromagnetic Wave Theory, Energy Harvesting, Signal Processing Technique, and Ultrasound Non-Distractive Testing.

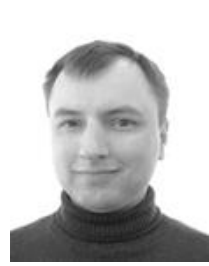

VALDAS JANKUNAS was born Lithuania, Klaipeda city, in 1976. In 2000-2003, he studied at Klaipeda University and completed the Vehicles bachelor's degree in engineering. In 2003-2005, he studied at Klaipeda University and acquired the electrical engineering master's degree. He received Ph.D. degree in Electrical and Electronics Engineering from Kaunas Technology University, in 2013. His current research interests include Energy Harvesting and Power Electronics. 


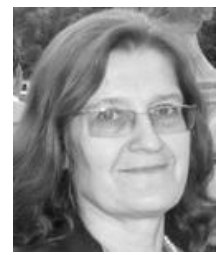

ELEONORA GUSEINOVIENE was born in Russia, has studied at Kaunas Polytechnic Institute and received qualification of electrical engineer, specialization "Electrical Drives and Automatics of Industrial Equipment" (cum laude). The PhD degree in Electrical and Electronics engineering she received in Kaunas Technology University in 2002. Currently, she is Professor, Head of Electrical Engineering Department and Senior Researcher at Klaipeda University. Research area: Special Electrical Machines, Electromagnetic Wave Theory, and Renewable Energy.

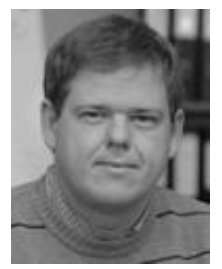

AUDRIUS SENULIS was born in Klaipeda, Lithuania, in 1979. He received the B.Sc. and M.Sc. degrees in Electrical engineering in Electrical Engineering Department of Klaipeda University in 2002 and 2004 respectively. The PhD degree he received in Electrical and Electronics engineering in Kaunas Technology University in 2013. Currently, he is a Assoc. Prof. in Electrical Engineering Department and Researcher at Klaipeda University. Also the engineer-constructor at JSC "Vejo projektai" in Lithuania. His research interests are: Special Oscillating Electrical Drives and Machines, Electromagnetic Wave Theory, Electrical Vehicles.

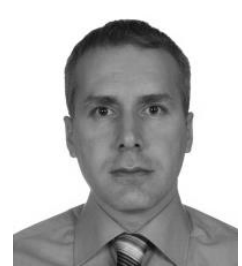

TAHIR CETIN AKINCI was born in Pınarbası, Turkey. He graduated from Electrical Engineering department at Klaipeda University. He received M.Sc. and $\mathrm{Ph} . \mathrm{D}$. degrees from Marmara University of Istanbul, Turkey, in 2002 and 2009 respectively. He received Assoc. Prof. degree in Electrical and Electronics Engineering from High Education Council of Turkey in 2014. His research interests are signal processing, electrical power systems, non-linear dynamical systems, soft computing and condition monitoring techniques. 